Gut, 1982, 23, 235-238

\title{
Correlation between pancreatic polypeptide response to secretin and ERCP findings in chronic pancreatitis
}

\author{
I STERN, I C ROBERTS-THOMSON, AND J HANSKY* \\ From Monash University Department of Medicine, Prince Henry's Hospital, and the Department of \\ Gastroenterology, The Royal Melbourne Hospital, Melbourne, Australia
}

SUMmaRY Previous studies have shown an impaired pancreatic polypeptide response to secretin in most patients with advanced chronic pancreatitis, but the sensitivity of the investigation in milder degrees of chronic pancreatitis remains unclear. In the present study the pancreatic polypeptide response to secretin was evaluated in 32 patients categorised as having advanced, moderate, or minimal chronic pancreatitis on the basis of the degree of abnormality of the retrograde pancreatogram. The pancreatic polypeptide response was abnormal (peak:basal pancreatic polypeptide ratio $<5$ ) in 17 of 19 patients $(90 \%)$ with advanced or moderate chronic pancreatitis, but was normal in patients with minimal disease. The simple and non-invasive nature of this test makes it an attractive investigation for evaluation of the severity of pancreatic damage.

A diagnosis of chronic pancreatitis is currently based on clinical features together with the findings of plain radiographs of the abdomen, abdominal ultrasound, computerised axial tomography, pancreatic secretory studies or endoscopic retrograde cholangiopancreatography (ERCP). Apart from calcification on plain radiographs of the abdomen, the only established diagnostic tests are pancreatic secretory studies or ERCP. The latter investigations, however, are invasive, time consuming, and uncomfortable for the patient. ${ }^{1}$ We have developed a means of assessing pancreatic function by the measurement of pancreatic polypeptide levels in serum after the intravenous injection of secretin. ${ }^{23}$ Using this method we have shown that $90 \%$ of patients with advanced chronic pancreatitis have an abnormal response and that there is a strong correlation between the results of a pancreatic secretory study and the pancreatic polypeptide response to secretin. ${ }^{2}$ In the present study the sensitivity of this test has been evaluated in patients with chronic pancreatitis of varying severity as defined by the degree of abnormality of the retrograde pancreatogram.

\footnotetext{
*Address for communications: Dr J Hansky, Monash University Department of Medicine, Prince Henry's Hospital, St. Kilda Road, Melbourne, Victoria 3004, Australia.

Received for publication 17 August 1981
}

\section{Methods}

SUBJECTS

Thirty-two patients were categorised as having advanced, moderate, or minimal pancreatitis primarily on the basis of findings at ERCP, before evaluation of the pancreatic polypeptide response. There were 23 males and nine females, with an age range of 33 years to 70 years (mean 48 years). Relevant clinical details are shown in the Table. Twenty patients with an age range of 30-56 years (mean 47 years) constituted the control group. Ten of these patients were normal volunteers, and 10 were investigated for abdominal pain (five) or steatorrhoea (five). All of the latter 10 had a normal ERCP and all were subsequently diagnosed as having non-pancreatic disease. Informed consent was obtained and the project was approved by the Research Advisory Committee of Prince Henry's Hospital (April, 1979).

All patients had plain radiographs of the abdomen, faecal fat estimations (considered abnormal if more than $6 \mathrm{~g}$ of fat were excreted in 24 hours), and postprandial blood glucose estimation which was followed by an oral glucose tolerance test where indicated.

The severity of chronic pancreatitis was determined by the degree of abnormality of the retrograde pancreatogram. ${ }^{4}$ Ten patients categorised as having advanced chronic pancreatitis all had major abnormalities of the main pancreatic duct including multiple 
Table Comparison of patient groups

\begin{tabular}{|c|c|c|c|c|c|c|c|c|}
\hline & \multirow{2}{*}{$\begin{array}{l}\text { Total } \\
\text { number }\end{array}$} & \multirow{2}{*}{$\begin{array}{l}\text { Age } \\
(y r)\end{array}$} & \multirow{2}{*}{$\begin{array}{l}\text { Length of } \\
\text { History } \\
(y r)\end{array}$} & \multicolumn{5}{|c|}{ Patient numbers } \\
\hline & & & & $\begin{array}{l}\text { Alcohol } \\
\text { related }\end{array}$ & Calcifn. & $\begin{array}{l}\text { Steat. } \\
(<6 g \\
\text { faecal } \\
\text { fat/day })\end{array}$ & $\begin{array}{l}\text { Diabetes } \\
\text { mellitus }\end{array}$ & $\begin{array}{l}\text { Confirmed by } \\
\text { laparotomy } \\
\text { ( } \pm \text { pancreatic } \\
\text { biopsy) }\end{array}$ \\
\hline $\begin{array}{l}\text { Chronic pancreatitis } \\
\text { Advanced } \\
\text { Moderate } \\
\text { Minimal }\end{array}$ & $\begin{array}{r}10 \\
9 \\
13\end{array}$ & $\begin{array}{l}48 \cdot 2 \pm 3 \cdot 5 \\
46 \cdot 8 \pm 1 \cdot 5 \\
49 \cdot 4 \pm 2 \cdot 2\end{array}$ & $\begin{array}{r}12 \cdot 8 \pm 1 \cdot 8 \\
7 \cdot 6 \pm 1 \cdot 3 \\
7 \cdot 2 \pm 1 \cdot 1\end{array}$ & $\begin{array}{l}8 \\
8 \\
2\end{array}$ & $\begin{array}{l}6 \\
1 \\
-\end{array}$ & $\frac{4}{-}$ & $\frac{6}{-}$ & $\begin{array}{l}7 \\
4 \\
5\end{array}$ \\
\hline
\end{tabular}

strictures, areas of dilatation, and ductal filling defects. Of the nine patients with moderate chronic pancreatitis, all had a single stricture of the main pancreatic duct in the head or body of the pancreas with dilatation of proximal ducts. All 13 patients with minimal chronic pancreatitis had dilatation, irregularity, and obstruction of side-branches only.

\section{TECHNIQUES}

After an overnight fast an indwelling catheter was inserted into the antecubital vein and kept patent with repeated saline flushing. A rapid intravenous injection of 2 units $/ \mathrm{kg}$ of Boots secretin was given and blood was taken 30 and 15 minutes before and at times $0,5,10,15$, $20,30,45$, and 60 minutes after injection. An aliquot of blood taken before secretin injection was used for glucose estimation by the glucose oxidase method to confirm euglycaemia in all patients before secretin injection. Sera were coded, stored at $-20^{\circ} \mathrm{C}$, and assayed 'blind' by a sensitive and specific radioimmunoassay (RIA) which has been previously described. ${ }^{8}$

\section{STATISTICS}

All values are expressed as mean \pm SEM. The significance of difference between unpaired means was tested by Student's $t$ test. P values of $<0.05$ were considered significant.

\section{Results}

Basal pancreatic polypeptide levels were similar in all groups; mean ( \pm SEM) levels being $13.6 \pm 2.5 \mathrm{pmol} / 1$ in control subjects and $15 \cdot 5 \pm 4 \cdot 6,22.0 \pm 11 \cdot 6$, and $11.8 \pm 2.7 \mathrm{pmol} / 1$ in patients with minimal, moderate, and advanced chronic pancreatitis respectively. All groups showed increases in serum pancreatic polypeptide levels after secretin injection with peak levels at five minutes in most individuals and at the latest by 15 minutes. However, mean peak pancreatic polypeptide levels were significantly lower in patients with advanced $(P<0.005)$ or moderate $(P<0.01)$ chronic pancreatitis than in control subjects (Fig. 1). Patients with minimal chronic pancreatitis had similar mean peak levels to controls.
When the pancreatic polypeptide response for each individual was calculated as a peak:basal pancreatic polypeptide ratio, similar results were seen (Fig. 2). The ratio was significantly lower in patients with advanced $(\mathrm{P}<0.005)$ or moderate $(\mathrm{P}<0.01)$ chronic pancreatitis than in control subjects. Previous studies in 50 patients with documented advanced chronic pancreatitis and 33 controls showed that $90 \%$ of chronic pancreatitis patients had a peak:basal pancreatic polypeptide ratio of less than 5 , whereas $91 \%$ of controls had a peak:basal ratio of greater than $5 .^{23}$ Pancreatic polypeptide levels rise with age ${ }^{9}$ and for this reason absolute levels, whether basal or stimulated, are of little value in the assessment of chronic pancreatitis. However, the peak:basal pancreatic polypeptide ratio appears a better index of pancreatic function and the lower limit of

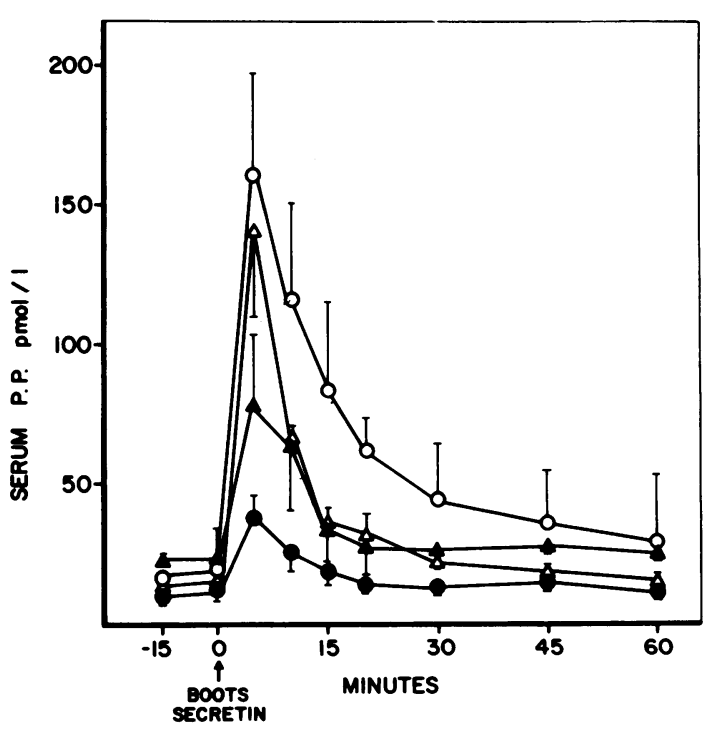

Fig. 1 Pancreatic polypeptide in the fasting state and after $2 U / k g$ intravenous Boots secretin in 10 patients with advanced chronic pancreatitis $(\Theta)$, nine patients with moderate chronic pancreatitis $(\Delta), 13$ patients with minimal chronic pancreatitis $(\triangle)$, and 20 control patients $(O)$. All values are mean $\pm S E M$. 


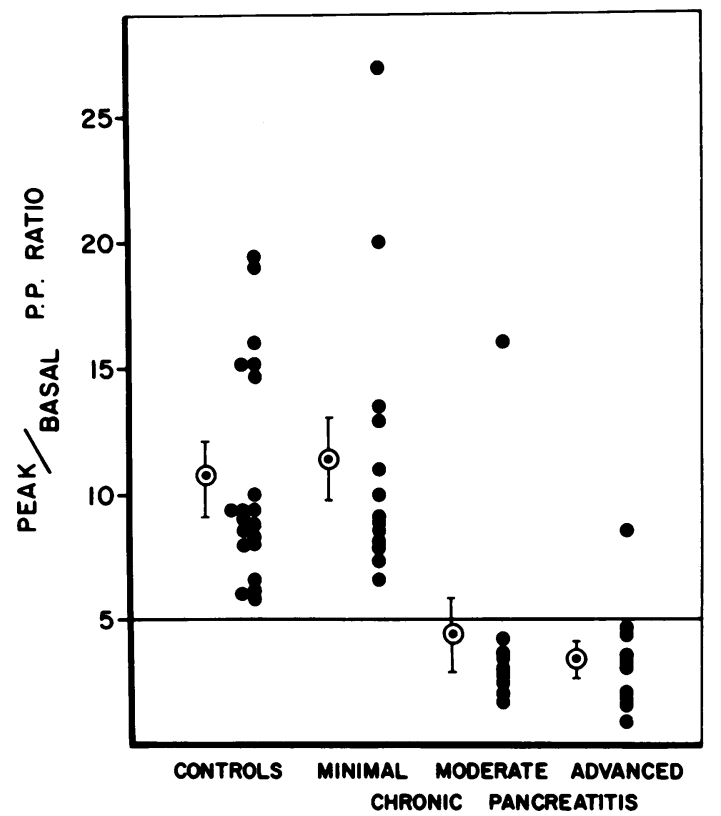

Fig. 2 Peak/basal pancreatic polypeptide in response to Boots secretin in 10 patients with advanced chronic pancreatitis, nine patients with moderate chronic pancreatitis, 13 patients with minimal chronic pancreatitis, and 20 controls. Each point represents the value for an individual patient, the circles and bars are the means \pm $S E M$ for each group as a whole.

normal has been defined as 5 . In the present study an abnormal pancreatic polypeptide response was present in nine of 10 patients categorised as having advanced chronic pancreatitis and in eight of nine patients categorised as having moderate chronic pancreatitis. All patients with minimal chronic pancreatitis and all controls had a normal peak:basal pancreatic polypeptide ratio.

\section{Discussion}

The results of this study confirm previous observations $s^{2}$ of impaired secretin-stimulated pancreatic polypeptide release in chronic pancreatitis. These observations suggest a loss of functioning pancreatic polypeptide cells which is consistent with a generalised destructive and fibrosing process and imply that the magnitude of the pancreatic polypeptide response may be related to the severity of the disease.

Previous studies in chronic pancreatitis have been performed using a protein-rich meal ${ }^{10}{ }^{11}$ or insulininduced hypoglycaemia ${ }^{12}$ as the stimulus of pancreatic polypeptide release. The pancreatic polypeptide response to food was a poor means of differentiating patients with chronic pancreatitis from control subjects, while additional studies are necessary to define the value of the response induced by hypoglycaemia. Previous studies by others have shown that pure (GIH) secretin does not release pancreatic polypeptide when given by slow infusion; ${ }^{13}{ }^{14}$ when given as a bolus injection, however, both forms of secretin have an equivalent pancreatic polypeptide stimulatory effect. ${ }^{15}$ The lack of effect of secretin infusion may be due to plastic absorbtion, of secretin by the infusion apparatus. ${ }^{16}$ The response to either form of secretin was shown to be reproducible, reliable, and dependent on extravagal mediation.

A previous study ${ }^{2}$ showed a peak:basal pancreatic polypeptide ratio of less than 5 in $90 \%$ of patients with advanced chronic pancreatitis and the present study was designed to evaluate the sensitivity of this test in milder forms of the disease. The degree of abnormality of the retrograde pancreatogram was chosen as the criterion for categorisation of patients and was validated, in part, by the presence of other features such as pancreatic calcification, malabsorption, and diabetes mellitus as shown in the Table.

The pancreatic polypeptide response was abnormal in 17 of 19 patients $(90 \%)$ with moderate or advanced chronic pancreatitis but was normal in all patients with minimal disease. Two patients with established chronic pancreatitis had a normal pancreatic polypeptide response, presumably indicating relative sparing of pancreatic polypeptide cells. Both patients had chronic pancreatitis related to alcohol abuse and one had pancreatic calcification on plain radiographs of the abdomen. The data did not support the possibility of greater destruction of polypeptide cells in alcoholic pancreatitis than in idiopathic or gallstone pancreatitis in those patients with moderate or advanced disease (although only three non-alcoholic patients were studied). The reasons for a false negative rate of $10 \%$ remains unclear but similar findings have been observed with standard pancreatic secretory studies including Lundh tests. ${ }^{17} 18$

The advantage of this test over standard pancreatic function tests is that it is quickly done and not uncomfortable for the patient. All patients responded with a peak pancreatic polypeptide level usually at five minutes - and, at the latest, at 15 minutes - after injection of secretin. Thus this test need take no more than half an hour and involves only the injection of secretin and the drawing of blood samples by a trained nursing sister. It does not demand highly trained staff, radiology facilities and screening time, is not invasive, and is well accepted by patients. Radioimmunoassay facilities are available in most major centres - the pancreatic polypeptide radioimmunoassay being simple and easily performed. 
The present study, showing an abnormal pancreatic polypeptide response in $90 \%$ of patients with moderate or advanced chronic pancreatitis at ERCP, corroborates our previous studies ${ }^{2}{ }^{3}$ in which an abnormal response was found in $90 \%$ of patients with an abnormal standard secretory test. Furthermore, preliminary studies suggest that an abnormal panereatic polypeptide response is specific for chronic pancreatitis; the response being normal in patients who have undergone vagotomy ${ }^{15}$ (and unpublished observation) and in most patients with pancreatic cancer (unpublished observation). Although other diseases will need to be looked at, it seems likely that the pancreatic polypeptide response to secretin may be an attractive alternative investigation for evaluation of the severity of pancreatic damage.

We are indebted to Dr Ronald Chance of Lilly Laboratories, Indianapolis, for the generous gifts of HPP antiserum, BPP and HPP standards. Thanks are also due to Sister K Deaton for help with blood collection and Miss J Waugh for technical assistance.

\section{References}

1 Waller SL. The diagnosis of exocrine pancreatic disease: the present position reviewed. Aust NZ J Med 1980; 10:351-62.

2 Stern AI, Hansky J, Korman MG. Pancreatic polypeptide after secretin: a new test for chronic pancreatitis. Gastroenterology 1980; 78:1270.

3 Stern AI, Hansky J. Secretin stimulated pancreatic polypeptide: a test for chronic pancreatitis. Aust $N Z J$ Med $1981 ; 11: 351-4$.

4 Kasugai K, Kuno N, Kizu M, Kobayashi S, Hattori K. Endoscopic pancreato-cholangiography II. The pathological endoscopic pancreato-cholangiogram. Gastroenterology 1972; 63:227-34.

5 Nakano S, Horiguchi Y, Takeda T, Suzuki T, Nakajima
S. Comparative diagnostic value of endoscopic pancreatography and pancreatic function tests. Scand J Gastroenterol 1974; 9:383-90.

6 Bohrman CA, Silvis SE, Vennes JA. Evaluation of the endoscopic pancreatogram. Radiology 1974; 113:297-304.

7 Roberts-Thomson IC. Endoscopic retrograde pancreatography. Analysis of the normal pancreatogram and changes which are associated with chronic pancreatitis and pancreatic cancer. Med J Aust 1977; 2:793-6.

8 Stern AI, Hansky J, Korman MG, Coupland G, Waugh J. Pancreatic polypeptide: studies in duodenal ulcer and after gastric surgery. Dig Dis Sci 1980; 25:485-8.

9 Floyd JC, Fajans SS, Pek S. A newly recognised pancreatic polypeptide: plasma levels in health and disease. Recent Prog Horm Res 1977; 33:510-70.

10 Adrian TE, Besterman HS, Mallinson CN, Garalotis C, Bloom SR. Impaired pancreatic polypeptide release in chronic pancreatitis with steatorrhoea. Gut 1979; 20:88-101.

11 Valenzuela JE, Taylor IL, Walsh JH. Pancreatic polypeptide response in patients with chronic pancreatitis. Dig Dis Sci 1979; 24:862-4.

12 Sive A, Vinik AI, Van Tonder S, Lund A. Impaired pancreatic polypeptide secretion in chronic pancreatitis. $J$ Clin Endocrinol Metab 1978; 47:556-9.

13 Adrian TE, Bloom SR, Besterman HS. PP physiology and pathology. In: Bloom SR, ed. Gut hormones. Edinburgh, Churchill Livingstone: 1978; 254-60.

14 Schwartz TW, Rehfeld JW. Mechanisms of pancreatic polypeptide release. Lancet 1977; 697.

15 Glaser B, Vinik AI, Sive AA, Floyd JC. Plasma human pancreatic polypeptide responses to administered secretin: effects of surgical vagotomy cholinergic blockade and chronic pancreatitis. J Clin Endocrinol Metab 1980; 50:1094-9.

16 Bitar KN, Zfass AM, Makhlouf GM. Binding of secretin to plastic surfaces. Gastroenterology 1978; 75:1080.

17 Dreiling DA. Pancreatic secretory testing in 1974. Gut 1975; 16:653-7.

18 Waller SL. The Lundh test in the diagnosis of pancreatic disease. Gut 1975; 16:657-8. 